\title{
Publisher Correction: Open Science principles for accelerating trait-based science across the Tree of Life
}

Rachael V. Gallagher (D), Daniel S. Falster (D), Brian S. Maitner(D), Roberto Salguero-Gómez, Vigdis Vandvik (D),

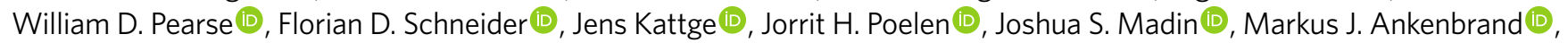

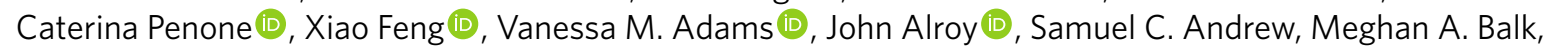
Lucie M. Bland, Brad L. Boyle, Catherine H. Bravo-Avila (D), Ian Brennan (1), Alexandra J. R. Carthey (D), Renee Catullo, Brittany R. Cavazos, Dalia A. Conde (1), Steven L. Chown (D), Belen Fadrique, Heloise Gibb, Aud H. Halbritter, Jennifer Hammock, J. Aaron Hogan, Hamish Holewa (D), Michael Hope, Colleen M. Iversen, Malte Jochum (1), Michael Kearney, Alexander Keller (D), Paula Mabee, Peter Manning (iD, Luke McCormack, Sean T. Michaletz (D), Daniel S. Park @, Timothy M. Perez, Silvia Pineda-Munoz, Courtenay A. Ray, Maurizio Rossetto, Hervé Sauquet (D, Benjamin Sparrow, Marko J. Spasojevic, Richard J. Telford (D), Joseph A. Tobias (1D, Cyrille Violle, Ramona Walls, Katherine C. B. Weiss (1), Mark Westoby (D), lan J. Wright (1) and Brian J. Enquist

Correction to: Nature Ecology \& Evolution https://doi.org/10.1038/s41559-020-1109-6, published online 17 February 2020.

In the version of this Perspective originally published, the first author of reference 39 was incorrectly listed as W. Cornwell and the publication year was incorrect. The reference should have read as follows: "Flores-Moreno, H. et al. fungaltraits aka fun fun : a dynamic functional trait database for the world's fungi (GitHub, 2019); https://doi.org/10.5281/zenodo.1216257". This has now been corrected.

Published online: 9 March 2020

https://doi.org/10.1038/s41559-020-1167-9

๑) Springer Nature Limited 2020 